\title{
Publication Ethics
}

In-Jae Lee

Department of Ethics Education, Seoul National University of Education, Seoul, Korea

Corresponding author: In-Jae Lee, Department of Ethics Education, Seoul National University of Education, 96 Seochojoongang-ro, Seocho-gu, Seoul 06639, Korea

Tel.: +82 234752411

Fax: +82 234752032

Email: ijlee@snue.ac.kr

Received March 9, 2017

Revised May 2, 2017

Accepted May 4, 2017

Published June 30, 2017

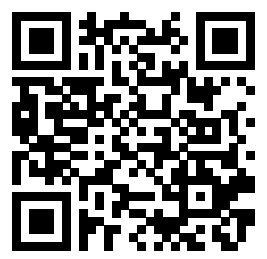

\begin{abstract}
After examining the values and core principles that are emphasized in publication ethics, I explored the international trends of recent publication ethics on the rules and ethical standards that the stakeholder of publication should obey. I discussed the core principles of publication ethics and the responsibilities of researchers and journal editors and publishers in publishing process, focusing on the Singapore Statement, the guide of publication ethics of Committee on Publication Ethics (COPE), and Elsevier, which are well-known international standards for publication ethics. Related topics discussed in publication ethics as an important area of research ethics include data fabrication and falsification, plagiarism, illegitimate authorship, duplicate publication, and conflict of interest. Important values that are emphasized in publication ethics are integrity, transparency, and trust. The following principles and behavior are required for researchers in publication ethics. First, researchers must be accurate and honest when presenting their research results in journals, proceedings, theses, and books. Second, authorship should be given to those who substantially contribute to the research. Third, if a violation of research ethics is recognized even after the research results are published, the research result should be withdrawn in whole or in part.
\end{abstract}

Keywords: Publication ethics, Plagiarism, Duplicate publication, Authorship, Conflicts of interest

\section{Introduction}

일반적으로 연구윤리(research ethics)는 연구자가 연구계획의 수립, 수행, 연구결과의 보고 과정에서 알고 실천해야 할 가치, 규 칙, 규범을 말한다. 다시 말해 연구윤리는 연구자가 정직하고 정확 하며, 성실한 태도로 바람직하고 책임 있는 연구를 수행하기 위해 지켜야 할 윤리적 원칙이나 행동 양식이라고 말할 수 있는 데(Lee, 2015), 연구 데이터나 결과물을 위조, 변조, 허위 발표를 하지 않 아야 하며, 자료의 수집과 기록, 분석과 번역에서의 편견, 왜곡, 과 장, 실수를 피해야 하는 등 정직성과 책임성의 가치가 핵심이 되고 있다. 출판윤리(publication ethics)는 연구성과를 검증 받아 알리고 널리 확산하기 위해 논문 작성, 학술 세미나에서의 발표, 논문 및 단행본 출판, 동료 심사(peer review) 등에서 지켜야 할 윤리적 기 준(ethical standards)을 의미한다. 즉, 출판윤리란 저자, 편집자, 동료 심사자, 출판사, 학계 및 연구기관이 수행해야 할 적절한 행위 를 기술하는 불문율의 성격을 가진 것으로부터 나온 일련의 원리나 규칙을 말한다(Caelleigh, 2003).
최근 연구윤리에서 출판윤리는 연구 진실성(research integrity) 과 관련하여 활발히 논의되고 있다. 이러한 논의에서는 특히 표 절(plagiarism), 데이터의 위조(fabrication), 이중게재(duplicate publication), 저자자격(authorship)과 같은 주제와 관련하여 과학 적 또는 학문적 규범을 위반하였는가가 핵심이 되고 있다. 출판윤리 와 관련한 구체적인 사안에 대한 관심이 증가하면서 윤리적인 출판 관행을 증진하기 위한 목적을 가진 몇몇 기관이 설립되었다. 이들 기관 중에서 널리 알려진 것은 영국에 본부를 둔 출판윤리협회 $(\mathrm{COPE})$ 와 국제의학학술지편집인협회(International Committee of Medical Journal Editors, ICMJE)가 대표적인데, 특히, 저자자격에 대한 $\mathrm{ICMJE}$ 의 기준은 다른 학문 공동체에서도 널리 채택되어 왔다.

표절, 위조, 이중게재, 부당한 저자자격의 관행(questionable authorship)에 이르기까지 출판에서의 부정행위는 크게 두 가지 측 면이 관련된다고 볼 수 있다. 하나는 연구자에게 가해지는 많은 논 문을 출판해야 한다는 압력이고, 다른 하나는 사기업에 의한 상업 적 압력이라고 할 수 있다. 전자는 학계의 치열한 경쟁의 문화 속에 서 '논문을 많이 출판하지 못하면 퇴출될 것이다(publish or perish)' 
라는 연구자에게 가해지는 압력과 관련하여 자주 설명되곤 한다. 이와 같이 연구자에게 가해지는 "publish or perish'의 압력은 연구 자들로 하여금 경쟁자들보다 우위에 서기 위해 이해충돌(conflicts of interest)을 숨기기, 출판에서의 편견(publication bias), 데이터 의 미공개(withholding of data), 이중게재, 논문 쪼개기(salami slicing), 과학 논문을 입맛대로 만들려고 하는 옳지 못한 행동 등을 하도록 유혹하곤 한다. 후자는 어떤 연구자가 특정 제약 회사에게 유리한 결과를 반영하는 논문을 출판하도록 상업적 압력을 받게 될 때를 말하는데, 이때에도 종종 출판윤리를 위반하는 중요한 원인 중의 하나가 되곤 한다. 그러므로 출판윤리를 규제하거나 증진하려 는 노력들은 개별 연구자들로 하여금 진실성에 부합하게 연구하도 록 촉진하는 도덕적인 분위기(moralistic tone)를 강조하거나 학술 연구와 가장 직접적으로 관련된 기관들(학술단체, 대학, 대학 병원) 이 출판의 관행을 규제하는 역할에 초점을 두기도 한다.

출판윤리는 학문 공동체와 출판 공동체에서 공통적으로 관심을 갖고 있는 중요한 연구윤리의 주제 중의 하나이다. 그렇기 때문에 특히 수많은 출판의 전문 기관들은 출판에 대한 윤리적 가이드라인 을 만들어 이를 따르도록 권장해 오고 있다. 연구윤리에서나 출판 윤리에서 모두 강조하고 있는 것은 책임있는 연구 수행(responsible conduct of research, RCR)을 위한 여러 가치나 바람직한 행동 양 식이며, 이 중에서도 단연 연구의 진실성(integrity)의 확보가 핵심 이라고 할 수 있다. 출판윤리는 객관적이고 진실한 연구 수행과 그 올바른 결과를 발표하는 것에 초점을 두기 때문에 연구윤리에서와 마찬가지로 진실성, 책임성, 신뢰성 등을 강조한다. 물론 연구결과 를 출판하는 활동과 관련된 당사자들, 즉 연구자, 편집인, 출판사, 동료 심사자, 연구비 지원 기관 등에 따라 맡은 역할이나 책임의 내
용이 다소 차이가 있지만, 서로 간의 신뢰를 바탕으로 각자에게 부 여된 윤리적 기준을 위반하지 않아야 한다는 점에서는 동일하다. 따라서 출판윤리는 연구윤리의 중요한 영역으로서 연구윤리에 포 함되는 개념이라고 말할 수 있으며, 출판윤리에서 쟁점이 되고 있 는 주요 내용은 연구윤리의 관심 사항과 상당 부분이 겹친다고 할 수 있다. 이하에서는 출판윤리가 추구하는 가치와 원리를 살펴본 후, 출판과 관련된 각 주체들이 지켜야 할 규칙이나 윤리적 기준에 대해 최근 국외 동향을 중심으로 살펴보고자 한다.

\section{Publication ethics}

\section{1. 출판윤리의 핵심 원리}

출판윤리에서 논의되고 있는 관련 주제는 데이터의 위조, 변조, 표절, 부당한 저자표시, 이중게재, 이해충돌 등 다양하며 이 각각에 서 강조하는 가치나 판단 기준도 다소 차이가 있을 수 있다. 그렇지 만 이 여러 주제별 쟁점에서 연구자에게 공통적으로 요구되는 핵심 원리나 가치들이 있다면 진실성(integrity), 투명성(transparency), 신뢰(trust)로 요약해 볼 수 있다. 그리고 출판윤리에서 이러한 핵 심 원리나 가치의 실천 주체는 연구자와 연구결과를 출판하는 편집 인과 출판사가 대표적이다. 이러한 출판윤리의 주체별 준수해야 할 가치와 행동 원리는 2010년에 발표된 싱가포르 선언(the Singapore Statement on Research Integrity; Singapore Statement, 2010) 이나 전 세계적으로 대표적인 학술지 출판사들(Nature, Science, Blackwell, Sage, Elsevier 등)에서 제시하고 있는 출판윤리의 가이 드라인에서 쉽게 찾아볼 수 있다.

\section{Table 1. Responsibilities}

\begin{tabular}{|c|c|}
\hline Research methods & $\begin{array}{l}\text { Researchers should employ appropriate research methods, base conclusions on critical analysis of the } \\
\text { evidence and report findings and interpretations fully and objectively. }\end{array}$ \\
\hline Research records & $\begin{array}{l}\text { Researchers should keep clear, accurate records of all research in ways that will allow verification and } \\
\text { replication of their work by others. }\end{array}$ \\
\hline Research findings & $\begin{array}{l}\text { Researchers should share data and findings openly and promptly, as soon as they have had an opportunity to } \\
\text { establish priority and ownership claims. }\end{array}$ \\
\hline Authorship & $\begin{array}{l}\text { Researchers should take responsibility for their contributions to all publications, funding applications, reports } \\
\text { and other representations of their research. Lists of authors should include all those and only those who meet } \\
\text { applicable authorship criteria. }\end{array}$ \\
\hline $\begin{array}{l}\text { Publication } \\
\text { acknowledgement }\end{array}$ & $\begin{array}{l}\text { Researchers should acknowledge in publications the names and roles of those who made significant } \\
\text { contributions to the research, including writers, funders, sponsors, and others, but do not meet authorship } \\
\text { criteria. }\end{array}$ \\
\hline Peer review & $\begin{array}{l}\text { Researchers should provide fair, prompt and rigorous evaluations and respect confidentiality when reviewing } \\
\text { others' work. }\end{array}$ \\
\hline Conflict of interest & $\begin{array}{l}\text { Researchers should disclose financial and other conflicts of interest that could compromise the trustworthiness of } \\
\text { their work in research proposals, publications and public communications as well as in all review activities. }\end{array}$ \\
\hline $\begin{array}{l}\text { Responding to irresponsible } \\
\text { research practices }\end{array}$ & $\begin{array}{l}\text { Research institutions, as well as journals, professional organizations and agencies that have commitments } \\
\text { to research, should have procedures for responding to allegations of misconduct and other irresponsible } \\
\text { research practices and for protecting those who report such behavior in good faith. When misconduct or } \\
\text { other irresponsible research practice is confirmed, appropriate actions should be taken promptly, including } \\
\text { correcting the research record. }\end{array}$ \\
\hline
\end{tabular}


첫째, 진실성이란 연구자는 자신이 제시하는 모든 연구방법이나 결과가 거짓, 편견, 선입견, 과장, 왜곡, 실수에 의한 것이 아니라 객관적인 사실에 입각하여 정직하게 보고되도록 해야 할 책임이 있 다는 것을 말한다. 싱가포르 선언을 보면, 출판윤리에서 강조하는 진실성과 관련하여 연구방법, 연구기록, 연구결과, 저자자격, 출판 물에서의 감사 표시, 동료 심사, 이해충돌, 부적절한 연구에 대한 보고 등에서 연구자가 지켜야 할 책임을 강조하고 있는데 이를 구 체적으로 제시하면 다음과 같다(Table 1).

둘째, 투명성이란 해당 연구나 출판을 위해 지원 받은 내용에 대 하여 정직하게 공개하는 것을 말한다. 즉, 연구결과에 관심을 가진 사람들은 이 연구결과 또는 연구출판이 누구의 지원에 의해 수행 되었는지를 알 권리가 있으므로 연구비 지원 사항에 대하여 논문 을 포함한 모든 결과물에 밝혀야 한다. 사기업, 자선단체, 정부 등 의 지원을 받아 이루어진 어떤 형태의 출판이든(연구 논문, 리뷰 논 문, editorials, commentaries) 지원받은 사항에 대해 출처를 항상 밝혀야 한다. 연구비를 지원하는 주체뿐만 아니라 연구와 출판에 기 여한 모든 사람들의 역할(연구의 설계, 실험 및 연구원 충원, 데이터 수집과 분석, 초고의 작성 또는 출판의 결정 등)도 만일 이러한 것들 이 저자나 기여자의 목록에서 분명하게 언급되지 않았다면 제시하 여야 한다. 출판윤리에서의 투명성 확보를 위해서는 연구자뿐만 아 니라 학술지의 편집자들도 출판 매뉴얼에 이와 관련된 출판 정책을 명시함으로써 연구자가 이를 알고 준수할 수 있도록 하여야 한다.

셋째, 신뢰란 해당 연구가 정상적인 과정을 거쳐 제대로 출판되 기 위해 이에 관련된 주체들 간에 믿음을 가질 수 있도록 진실되 게 행동하는 것을 말한다. 학술지를 발간하는 출판사들(academic publishers)은 대체로 신뢰를 기반으로 하고 있다. 편집자들은 동료 심사자들이 공정한 평가를 할 것이라고 믿고, 투고 논문의 저자들 은 편집자들이 공정하게 동료 심사자를 배정할 것이라고 믿는다. 또 한 독자들은 동료 심사 과정이 믿을만하다고 생각한다. 학술지의 출 판은 충돌을 일으키기도 하고 지적인 측면과 재정적 또는 정치적 측 면의 강력한 이해관계 속에서 이루어지고 있다. 이러한 이해관계를 관리하기 위하여 고안된 훌륭한 결정과 강력한 편집 과정은 지속적 이고 효율적인 출판 시스템을 함양하며 그 결과로 학술 단체, 학술 지 편집자, 저자, 연구비 지원자, 독자 및 출판사에게 이익을 가져다 준다. 따라서 바람직한 출판의 문화는 우연히 발전하는 것이 아니라 적극적으로 증진될 때에만 확립될 수 있다(Graf et al., 2007).

\section{2. 출판윤리의 주체별 역할과 책임}

출판윤리와 관련된 주요 쟁점들을 출판윤리에서 가장 중요한 위 치를 차지하고 있는 연구자와 편집인 및 출판사의 책임에 초점을 두어 이들의 역할에 대하여 정리해 보면 다음과 같다.

1) 출판윤리에서 연구자의 책임(Lee, 2015)

연구결과의 발표 또는 출판은 연구계획의 수립이나 데이터의 수
집 및 분석과 함께 연구활동에서 중요한 과정 중의 하나이다. 학문 의 역사를 보면, 자신의 연구 성과물을 다른 연구자가 가로채지 못 하도록 하기 위해 공개하지 않고 비밀로 한 적도 있었지만, 오늘날 에는 동료 심사를 거쳐 논문을 게재하거나 학술 컨퍼런스 등을 통 해 공식적으로 발표되지 않은 연구의 결과는 그 업적을 제대로 인 정받지 못한다. 즉, 연구자가 자신이 수행한 연구결과를 발표하는 것은 그 연구결과가 널리 통용되고 객관적으로 평가 받을 수 있는 기본적인 조건이다.

연구결과를 발표하는 전형적인 형태 중의 하나가 동료 심사를 거 쳐 전문 학술지에 논문을 게재(출판)하는 것이다. 이 경우 연구자들 의 연구결과는 연구 논문(초고) 작성 및 투고, 동료 심사, 학술지 편 집인의 논문 게재 승인, 최종 학술지 게재 등의 과정을 통해 공식적 으로 인정을 받는다. 연구결과는 책이나 학술지 논문 등과 같은 서면 형식으로 보고되는 경우가 보통이지만 학술 컨퍼런스나 학술 회의 에서 구두 또는 포스터 발표 등으로도 이루어진다. 이때 인쇄된 발표 자료나 슬라이드 자료 등이 제시되기도 한다. 최근에는 인터넷 상에 연구결과를 발표하는 경우도 늘고 있다. 이렇게 연구자들의 연구결 과는 공개적이고 공식적인 검토를 거쳐 공유 및 확산되며, 이를 통해 자신 또는 다른 연구자의 후속 연구의 토대가 된다. 만일 한 연구가 타당한 방법에 의해 이루어졌는지, 데이터의 수집과 분석 및 해석이 객관적이고 타당한 것인지, 해당 연구성과를 이루기까지 편견이나 실수, 의도적인 조작, 이해충돌은 없어 공정하고 믿을만한 것인지, 그 결과가 해당 학문이나 사회의 발전에 유익한지 등을 충분히 검토 할 수 있는 과정이 없다면 그 연구성과를 누구도 신뢰할 수 없을 것 이다. 이러한 개방적이고 객관적인 논의 과정은 해당 연구 분야가 점 진적으로 발전해 가는데 필수적인 것이다. 그러므로 연구자는 자신 의 연구결과가 학문 공동체의 비판적인 검토를 통해 객관적으로 검 증 받아 학문 공동체나 사회 발전에 이바지하여야 한다는 책임감을 가지고 자신이 수행한 연구결과를 정직하고 공정하게 발표해야 할 의무가 있다.

연구자가 연구결과 발표에서의 지켜야 할 윤리적 기준은 무엇인가? 다시 말해 출판윤리에서 연구자에게 요구하는 원칙과 행동 양식은 무 엇인가? 한마디로 연구자가 자신의 연구결과를 학술지 논문, 학술대 회 발표 논문, 학위 논문, 저서 등으로 발표할 때 정확하고 거짓이 없 도록 하는 것이다. 이와 관련되는 주요 내용을 좀 더 구체적으로 살펴 보면 다음과 같다.

(1) 한국심리학회 연구윤리(2003. 08.) 제33조에 나타나 있는 것 처럼, 타인의 연구성과나 자료를 활용할 때는 정확한 출처와 인용 표시를 해야 하며, 마치 자신의 것처럼 도용해서는 안 된다.

(2) 자신의 이전 연구결과를 활용할 때도 정확한 출처를 밝혀 마치 처음 발표하는 것처럼 자신과 독자를 속이지 않아야 한다.

(3) 자신의 연구 업적을 부풀리기 위해 자신의 이전 연구와 중요한 내용이 같거나 실질적으로 유사한 내용을 출처표시 없이 마치 새 로운 것처럼 발표하지 않고 논문 쪼개기 등을 하지 않아야 한다. 
(4) 연구에 기여하지 않았으면서 저자로 이름을 올리거나, 저자로 서 정당한 자격이 있는 사람을 저자에서 배제하는 행위를 하지 않 아야 한다.

(5) 연구결과를 대중매체에 과장하여 공개하여서는 안 된다.

(6) 연구결과가 발표된 이후에도 연구윤리에 위반되는 사항을 인 지하게 될 경우에는 연구결과의 전부 또는 일부를 철회해야 한다.

논문의 출판은 연구자로서 인정받는데 필수적인 잣대로 작용하 기 때문에 연구자가 학술지에 논문을 발표할 때는 정확하여야 한 다. 즉, 일정한 자격을 갖춘 사람에게만 저자 자격(authorship)을 허용하고 실질적으로 기여한 정도에 따라 저자의 순서를 정함으 로써 공로(credit)를 합당하게 배분해야 한다. 즉, 대학원생이나 박 사 후 연구원과 같은 젊은 연구자들에 대해 정당하게 공로를 인정 해야 하며, 연구에 실질적으로 기여하지 않는 사람을 논문 저자로 올리는 이른바 '명예저자(honorary authorship)'의 관행을 따라 하 지 않는 것이 중요하다. 또한 같은 논문을 중복 발표하거나, 하나의 논문을 여러 편으로 나누어서 발표해서는 안 되며, 동료 심사(peer review)를 거치지 않은 연구성과를 일반 언론을 통해 국민들에게 직접 알려 대중적 명성이나 금전적 이익을 추구해서도 안 된다.

세계적으로 저명한 출판사인 Elsevier에서는 동시투고 (simultaneous submission), 다중게재(multiple publication), 이중 게재(duplicate publication)와 관련한 출판윤리의 가이드를 다음 과 같이 제시하고 있다(Elsevier, 2005). 즉, 연구자는 이전에 결 코 발표(게재)되지 않은 최초의 연구(original research)에 근거하 여 자신의 논문을 써야 할 의무가 있으므로 이중게재를 위해 의도 적으로 논문을 투고하거나 다시 투고하는 것은 출판윤리를 위반하 는 것으로 간주된다는 것이다. 여기서 동시투고란 연구자가 한 논 문을 동시에 2 개 이상의 다른 학술지에 투고함으로써 어떤 특정 한 논문이 하나 이상의 학술지에 게재되게 하는 것을 말하고, 이중 게재 또는 다중게재란 2 개 혹은 그 이상의 논문이 서로 출처를 밝 히지 않고 본질적으로 동일한 가설, 데이터, 논점, 결론을 공유하 는 것을 말한다. 이러한 이중게재의 유형은 문자 그대로 동일한 중 복(literal duplication), 부분적이지만 실질적인 중복(partial but substantial duplication), 말바꿔쓰기를 통한 중복(even duplication by paraphrasing)과 같이 다양한 방식으로 발생한다.

최초의 연구에 대한 이중게재가 비윤리적으로 간주되는 주요한 이유 중의 하나는 하나의 연구결과를 의도적으로 이중으로 계산하 거나 부적절하게 비중을 둠으로써 이용 가능한 증거를 왜곡하기 때 문이다. 출판윤리에서 가장 중요한 원칙은 출판을 위한 논문은 최 초의 것이어야 하며, 다른 어떤 곳에 투고되지 않아야 한다. 투고를 할 때 저자는 관련된 논문, 출판 중인 유사한 논문 또는 번역된 것 등 어떤 것이든 상세하게 알려야 한다. 이중게재의 경계선은 학문 분야마다 다르기 때문에 출판사는 논문 투고의 가이드를 제시해야 하며, 연구자는 투고하고자 하는 학술지의 투고 지침을 정확히 알 고 이를 준수하여야 한다.
2) 학술지 편집인 및 출판사의 책임

일반적으로 출판윤리에서는 연구자와 학술지를 발간하는 편집 인과 출판사의 연구윤리에 대한 관심과 실천력이 가장 중요하다고 본다. 앞에서 언급했듯이, 연구자가 알고 실천해야 할 출판윤리에 서의 가치나 규칙이 있을 뿐만 아니라 편집인이나 출판사에서도 정 해서 실천해야 할 몇 가지 중요한 원칙이나 규칙이 있다.

그렇다면 편집인과 출판사에는 무엇을 지향하고 있고 어떻게 이 를 실천해야 할까? 출판사들의 출판 관련 규칙을 제대로 아는 것은 연구자의 입장에서 보면 자신이 알고 따라야 할 출판윤리에서 제시 하고 있는 규칙의 상당 부분을 이해할 수 있는 것이다. 출판윤리에 서 주로 많이 논의되고 있는 데이터의 위조, 변조, 표절, 부당한 저 자표시, 이해충돌, 이중게재 등이 발생하지 않도록 하기 위해 연구 자와 학술지 편집인과 출판사는 함께 인식하고 지켜야 할 윤리적 기준을 공유하고 출판과정에서 서로 이를 준수할 수 있도록 노력해 야 한다. 특히 학술지의 편집인과 출판사에는 연구자들이 논문을 투고할 때 반드시 준수해야 할 이러한 출판윤리의 중요 사항을 출 판 매뉴얼 또는 출판 정책 등의 방식으로 명확하게 공지하고 이를 엄정하게 적용하여야 한다. 이하에서 이와 관련된 몇 가지 사항을 언급하면 다음과 같다.

첫째, 출판사는 연구자와 독자로부터 불만을 듣지 않도록 해야 한다. 특히 현재의 동료 심사 제도로는 연구자의 의도적인 사기 (fraud)를 찾아내기 어렵다. 동료 심사는 비용도 많이 들고, 편견이 개입되며 공정한 검토를 위해 충분하지 않을 수도 있다는 비판에 귀 기울이고 이를 예방할 수 있도록 동료 심사의 공정성과 객관성 을 확보할 수 있는 합리적인 방안을 마련하여야 한다.

둘째, 학술지 편집인들은 저자를 신뢰할 수 있어야 한다. 학술 지는 여러 방식으로 이중게재를 예방하기 위하여 노력하고 있지 만 실제로 이중게재에 대한 제제는 미약할 뿐이다. 그러므로 이중 게재를 예방하기 위한 적절한 조치가 필요한데, 우선 출판 가이드 라인을 투고자들이 명확히 인식하여 준수할 수 있도록 하여야 한 다. 이하에서는 자신의 이전 저작물의 재활용(text recycling)과 관 련하여 $\mathrm{COPE}$ 의 가이드라인을 소개하고자 한다(Committee on Publication Ethics, 2016).

$\mathrm{COPE}$ 는 다양한 학문 분야를 망라한 수많은 해외 저명 학술지의 편집인들을 대상으로 출판윤리에 대한 자문 및 교육을 하고 있다. 그러므로 여기서 제공하고 있는 중복게재 또는 텍스트 재활용에 대 한 지침은 $\mathrm{COPE}$ 회원 학술지의 편집인들이 투고 논문을 검토할 때 기준으로 활용하기 때문에, $\mathrm{COPE}$ 의 지침을 잘 이해한다면 연구자 가 투고하고자 하는 학술지의 중복게재 또는 텍스트 재활용에 대한 판단 기준에 어긋나지 않도록 하는데 도움이 될 것이다. 이하에서 는 연구자들이 자신의 연구 성과를 재활용함에 있어 유익한 참고가 된다고 생각하여 다소 길지만 $\mathrm{COPE}$ 가 제시한 텍스트 재활용에 대 한 가이드라인을 제시하고자 한다.

이 지침은 학술지 편집자들이 텍스트 재활용의 사례를 다루고 
자 할 때 참고할 수 있도록 하기 위한 것이다. 흔히 자기표절(self plagiarism)이라고 알려져 있는 텍스트 재활용은 동일한 텍스트의 어느 부분을 대개는 출처를 밝히지 않고 하나 이상의 학술지에 발 표하는 것을 말한다. 여기서 텍스트 재활용이라는 용어는 일반적 으로 적절하게 출처를 밝히지 않고 다른 사람의 단어나 아이디어를 사용했을 때 발생하는 “전형적인" 표절과 구분하기 위해 선택한 것 이다. 텍스트 재활용과 혼동하지 않도록 하기 위해 구분하는 문제 가 바로 중복게재(redundant publication) 또는 이중게재(duplicate publication)이다. 이 용어는 일반적으로 데이터나 아이디어가 반복 해서 출판되어 횔씬 더 큰 문제를 유발할 때를 의미하는데, 종종 최 소한 같은 저자 1 인이 포함되어 있다.

학술지의 편집인들은 텍스트 재활용을 어떻게 다루고 있을까? 편 집인들은 텍스트 재활용이라는 각각의 사례를 살필 때 중복되는 부 분이 얼마나 ‘중요한가’ (important)를 바탕으로 다음과 같은 몇 가지 요인들을 고려한다. 첫째, 얼마나 많은 텍스트가 재활용되고 있는 가? 둘째, 어디에 게재된 논문에서 텍스트 재활용이 일어나고 있는 가? 셋째, 재활용된 텍스트의 출처를 밝히고 있는가? 넷째, 재활용 되는 논문이 연구 논문(research article)인가 아니면 연구가 아닌 논 문(non-research article)인가? 다섯째, 저작권 침해가 있는가? 여섯 째, 논문이 출판된 시기와 장소에서 문화적 규범(기준)은 무엇인가?
텍스트 재활용은 투고된 초고 또는 출판된 논문에서 발생한다. 이 는 연구 논문, 리뷰 논문 등 여러 가지 다른 논문의 형태에서 발생할 수 있으며, 한 논문 내의 다른 부분에서도 발생할 수 있다. 중첩되는 중요한 부분이 두 논문 또는 그 이상의 논문에서 나타났을 때, 편집 인은 저자에게 그 이유에 대해 명료화를 요구하거나 일정한 조치를 고려하게 된다.

그렇다면 학술지 편집인들은 텍스트 재활용에 대해 검토할 때, 어 떤 요인을 중요하게 고려할까? 먼저 편집인들은 이전에 출판된 것과 중요하게 겹치는 부분이 있는지를 확인하고, 중복되는 정도가 내용 의 새로움(독창성)과 관련하여 학술지의 독자에게 얼마나 중요하게 영향을 미치는지를 보게 된다. 또한 논문의 저자가 정당하게 텍스트 재활용을 하고 있는지, 이미 게재(발표)한 아이디어나 데이터를 마치 새로운 것처럼 잘못 제시하고 있는지를 살펴본다. 텍스트 재활용과 관련하여 중요하게 중복된다는 것을 결정하는데 있어 고려해야 할 요인들에 대해서는 크게 연구 논문과 리뷰 논문(의견, commentary articles)로 나누어 살펴보면 다음과 같다.

(1) 연구 논문

연구 논문에서 텍스트 재활용에 대해 검토할 때 고려해야 할 요 인은 Table 2 와 같다.

\section{Table 2. Research articles}

\begin{tabular}{ll}
\hline Components of the article & Considerations when determining text recycling \\
& -Some degree of text recycling in the background/introduction section of an article may be unavoidable, \\
particularly if an article is one of several on a related topic. & -Duplication of background ideas may be considered less significant or even considered desirable, contrasted \\
with duplication of the hypothesis, which will only be appropriate in very closely related papers. & -Editors should consider how much text is repeated verbatim, and whether the original source is cited \\
(although editors should note that citing the source is not a justification per se). & -Use of similar or identical phrases in methods sections where there are limited ways to describe a method is \\
& not unusual; in fact text recycling may be unavoidable when using a technique that the author has described \\
before and it may actually be of value when a technique that is common to a number of papers is described. & -Editors should use their discretion and knowledge of the field when deciding how much text overlap is \\
acceptable in the methods section. & \\
-An important factor to consider is whether the authors have been transparent, stating that the methods have \\
already been described elsewhere and providing a citation.
\end{tabular}
already been described elsewhere and providing a citation.

-Text recycling is almost always unacceptable in the results section if it duplicates previously published data. In such situations, editors should consider whether this is a redundant (duplicate) publication. -Occasionally the authors may have legitimate reasons to include their previously published data, for example,

Results if they are reporting an extension of their previous research. In such cases, this duplication must always be reported transparently and be properly attributed and compliant with copyright requirements.

-The re-use of data without clear scientific justification and transparency should be dealt with according to COPE guidelines for redundant (duplicate) publication rather than as 'simple' text recycling.

Discussion -Some degree of text recycling may be acceptable in the discussion; however, as the majority of the discussion should focus on putting the results of the current study in context, large amounts of text recycling is unlikely to be acceptable, especially if previously published ideas are presented as new.

-Text recycling is unlikely to be acceptable in the conclusions of an article. If the conclusions contain recycled text, editors should consider whether the content of the article is novel.

-Reproduction of previously published figures or tables may represent data duplication if the authors do not provide a justification (see 'Results') and, if reproduced without permission, may result in copyright infringement.

COPE, Committee on Publication Ethics. 
(2) 의견, 리뷰 논문

의견, 리뷰 논문, 주석 논문과 같이 연구 논문이 아닌 경우에도 원칙상 연구 논문과 같은 지침을 지켜야 한다. 비록 연구 논문이 아 닐지라도 비판적이고 의견을 반영한 것이기에, 편집자들은 이미 출 판된 의견, 아이디어가 새롭게 더 발전되지 않고 이전에 출판된 것 으로부터 텍스트가 나왔다면 또는 이미 발표된 것에 대한 어떤 출 처도 밝히지 않고 새로운 것처럼 제시되었다면 이에 대한 설명을 요청하거나 적절한 조치를 취해야 한다.

셋째, 학술지 편집인들은 투고된 논문을 심사하는 과정에서 다 음과 같은 상황을 인지하게 되면 논문 철회, 우려 표명, 수정 등과 같은 조치(Table 3)를 취할 수 있는 바, 연구자의 입장에서는 이를 통해 자신의 논문이 출판되기 위해 출판윤리의 위반을 막기 위해 반드시 유의해야 할 점이 무엇인지를 파악하는데 도움을 받을 수 있을 것이다.

\section{Conclusion}

표절, 데이터 위조, 변조와 같은 출판에서의 부정행위는 정당한 학문적 행위로 인정받을 수 없을 정도로 심각한 연구윤리 위반일 뿐만 아니라 학회나 학술지 출판사로부터 제재를 받게 되는 비윤 리적인 연구 행위(unethical research behavior)의 형태로 인식되 고 있다. 뿐만 아니라 정직한 연구자와 편집인들은 중복게재와 같 은 비윤리적 행동이 연구를 왜곡하며, 정당화될 수 없는 저자 자격 (non legitimate authorship)처럼 훨씬 덜 나쁜 비행이라고 간주될 수 있는 빈약한 연구 실천의 형태라고 할지라도 연구에서의 '도덕 적 오염물질(moral pollutant)'의 형태라고 인식하고 있다. 그러므 로 $\mathrm{COPE}$ 의 출판윤리 정책에 의하면, "학술지 편집자들은 연구의 기록에서 진실성을 유지하고, 논문이 투고되거나 출판될 때 출판 부정행위를 파악할 수 있도록 최선의 노력을 다할 최고의 의무가 있다"고 주장한다.

연구자들이 출판윤리를 위반하는 경우는 다양하다. 치열한 경쟁
에서 우위에 서기 위해 다른 연구자보다 많은 연구 업적을 내야 하 는 상황에서 의도적으로 부정행위를 저지를 수도 있고 연구자로서 지켜야 할 출판윤리를 잘 모르거나 실수로 그럴 수도 있다. 그렇지 만 출판윤리가 확립되기 위해서는 무엇보다도 연구자가 출판윤리 의 중요성을 인식하고 연구자에게 요구되는 출판의 윤리적 기준을 잘 실천하여야 한다. 이러한 연구자의 높은 윤리의식의 함양 및 실 천과 함께 중요한 것은 바로 학술지의 편집인과 출판사가 연구자의 출판윤리의 실천을 지원할 수 있는 확고한 출판윤리 정책을 수립하 여 엄정하게 적용하는 것이다. 학술지의 편집인과 출판사는 연구 논문의 출판의 책임을 맡고 있는 입장에서 높은 수준의 출판윤리의 문화를 정립하는데 있어 연구자와 함께 중요한 양대 축을 이루고 있다. 그러므로 연구자와 학술지의 편집인 및 출판사의 출판윤리의 실천을 위한 상호 존중과 유기적인 협력이 필요하다.

양질의 연구 논문을 발굴하여 출판해야 할 중요한 역할을 담당 하고 있는 학술지 편집인과 출판사들은 먼저 출판에서의 연구부 정을 피하기 위한 자체의 명백한 출판윤리 정책(publication ethics policies)을 자율적으로 마련하여 시행하여야 한다. 이미 국내·외 의 학술지에서 적용하고 있는 것처럼, 출판윤리의 기본적인 가이드 라인에 "저자가 투고한 논문이 최초의 것(original)이며, 다른 곳에 출판을 고려하지 않았으며, 이전에 출판되지도 않았다."는 내용에 서약하도록 하는 것이 한 예라고 할 수 있다. 또한 부당한 저자표시 (illegitimate authorship)를 예방하기 위해 학술지는 저자들에게 저 자 목록에 있는 사람들이 저자로서의 자격을 갖추었는지를 확인하 기 위한 일정한 형식에 서명하도록 요구한다. 저자들 간의 분쟁을 막고 정당한 저자의 업적을 공정하게 배분하기 위해 수많은 학술지 들은 저자들이 해당 연구에서 어떻게 기여했는지를 증명하는 서명 을 요청하여야 한다. 다음으로 학술지는 투고된 논문의 독창성을 확보하기 위한 다양한 표절 및 중복게재 검색 프로그램을 사용하는 것도 필요하다. 보다 심각한 연구부정행위(데이터 위조나 변조 등) 는 대체로 동료 심사 과정에서 심사자들에 의해 드러나곤 한다. 이 러한 연구부정행위들은 필요한 검증을 위해 해당되는 기관에게 바 로 보고되어야 한다.

Table 3. Contents of retraction, expression of concern, correction

\begin{tabular}{ll}
\hline & -Journal editors have clear evidence that the findings are unreliable, either as a result of misconduct \\
(e.g. data fabrication) or honest error (e.g. miscalculation or experimental error). \\
Retracting a publication & -The findings have previously been published elsewhere without proper cross referencing, permission \\
& or justification (i.e. cases of redundant publication). \\
& -It constitutes plagiarism. \\
-It reports unethical research. & \\
-Journal editors receive inconclusive evidence of research or publication misconduct by the authors. & -There is evidence that the findings are unreliable but the authors' institution will not investigate the case. \\
Expression of concern & -They believe that an investigation into alleged misconduct related to the publication either has not been, \\
& or would not be, fair and impartial or conclusive. \\
Correction & -An investigation is underway but a judgement will not be available for a considerable time. \\
& -A small portion of an otherwise reliable publication proves to be misleading (especially because of honest error). \\
& -The author/contributor list is incorrect (i.e. a deserving author has been omitted or somebody who does not meet \\
& authorship criteria has been included). \\
\hline
\end{tabular}


매우 흔하게 발생하는 출판에서의 연구부정행위를 예방하고 어 떤 연구가 객관적으로 신뢰받을 수 있도록 하기 위해서는 일차적으 로 연구자(저자), 학술지 편집자, 동료 심사자가 출판과 관련하여 부여된 높은 윤리적 기준을 인식하고 서로 협력하여 준수해야 한 다. 지속적인 출판윤리가 필요한 이유가 바로 여기에 있다.

* 본 논문은 연구윤리정보센터(www.cre.or.kr)에서 발행하는 뉴 스레터 “研精” (Vol, 35, 2016년 12월, pp. 1-8)에 실린 필자의 글을 보완한 것임.

\section{References}

Caelleigh AS. Roles for scientific societies in promoting integrity in publication ethics. Science and Engineering Ethics, 9: 221-241, 2003.

Committee on Publication Ethics. Text recycling guidelines. 2016.

Elsevier. Simultaneous submission/multiple, duplicate publication. 2015.

Graf C, Wager E, Bowman A, Fiack S, Scott-Lichter D, Robinson A. Best practice guidelines on publication ethics: a publisher's perspective. International Journal of Clinical Practice, 61: 1-26, 2007.

Lee IJ. Understanding research ethics and ethical practice. Dongmunsa, Seoul, pp53-108, 2015.

Singapore Statement. Singapore statement on research integrity. 2010. 


\section{국문초록}

\section{출판윤리}

이인재

서울교육대학교 윤리교육과, 서울, 한국

출판윤리에서 강조하는 가치와 핵심 원리를 살펴본 후, 출판과 관련된 각 주체들이 지켜야 할 규칙이나 윤리적 기준에 대해 최근 출판윤리의 국제적 동향을 탐구하고자 하였다. 출판윤리에 관한 국제적 표준을 잘 제시하고 있는 싱가포르 선언, Committee on Publication Ethics (COPE)와 Elsevier의 가이드를 중심으로 출판윤리의 핵심 원리, 출판윤리에서의 연구자와 학술지 편집인과 출판 사의 책임이 무엇인지에 대해 논의하였다. 연구윤리의 중요한 영역으로서 출판윤리에서 논의되고 있는 관련 주제는 데이터의 위조, 변조, 표절, 부당한 저자표시, 이중게재, 이해충돌 등 다양하다. 출판윤리에서 강조하는 중요한 가치에는 진실성, 투명성, 신뢰이다. 출판윤리에서 연구자에게 요구하는 원칙과 행동 양식은 다음과 같다. 첫째, 연구자가 자신의 연구결과를 학술지 논문, 학술대회 발 표 논문, 학위 논문, 저서 등으로 발표할 때 정확하고 정직해야 한다. 둘째, 연구에 실질적으로 기여한 사람에게 저자자격을 부여해 야 한다. 셋째, 연구결과가 발표된 이후에도 연구윤리를 위반한 사항을 인지하게 되면 연구결과의 전부 혹은 일부를 철회해야 한다.

핵심어: 출판윤리, 표절, 이중게재, 저자자격, 이해상충

\section{참고문헌}

이인재. 연구윤리의 이해와 실천. 동문사, 서울, $\mathrm{pp} 53-108,2015$. 


\section{中文摘要}

\section{出版伦理}

李仁宰

首尔教育大学伦理教育科, 首尔, 韩国

在考察了出版伦理中所强调的价值观和核心原则之后，探讨出版伦理对出版者应遵循的规则和伦理标准最新国际趋势。 以包含出版伦理国际标准的新加坡声明, Committee on Publication Ethics（COPE）以及Elsevier指南为中心，探讨出版 伦理的核心原则, 出版伦理中的研究人员的责任以及期刊出版过程中编辑者和出版者的责任。出版伦理作为研究伦理的 重要领域, 其包括数据捏造和伪造, 抄袭, 非法署名, 重复出版, 利盆冲突等主题。在出版伦理学中强调的重要价值是 正直、透明和信任。出版伦理中研究者需要遵循以下原则和行为：首先, 研究人员应该把研究成果准确地、正直地发表 在期刊、学术大会发表论文、学位论文以及书籍当中。其次，研究中实际参与贡献的人员赋予作者资格。第三，即使研 究成果公布后，若发现违反研究伦理，需应该全部或部分撤回研究成果。

关键词: 出版伦理, 剽窃, 一稿两投, 作者资格, 利益冲突 